\section{Multiple sclerosis, viruses and glycolipids}

SIR-For many years now, and again recently in Nature (318, November 1985 p101, p104, p154), there has been speculation as to the role of viruses in multiple sclerosis (MS). MS is often considered to be an autoimmune disease initiated by infection; the question has always been and remains, which of the many viruses implicated causes the disease? One possibility, which has perhaps not received sufficient attention, is that MS could be caused not by just one virus but by many enveloped viruses acting together or individually.

All the viruses that have been implicated in MS are capable of infecting the nervous system, and all are enveloped viruses. The membrane of an enveloped virus reflects that of the cell from which it has budded. Indeed it has been suggested that such viruses may be able to trigger autoimmune reactions by the incorporation of host protein into their viral envelope but, with a few possible exceptions, the budding virus takes only the virallycoded proteins which have been inserted into the cell membrane, and the lipid molecules surrounding them. It is the host-cell derived lipid molecules that could be the key to the problem.

The lipid molecules of cell membranes have suffered for too long by being represented as the homogeneous tadpole-like molecules of the cell membrane. In fact they are far from being homogeneous in either their biochemistry or their distribution. Neither are these molecules immunologically inert, they are haptens and can under the right circumstances induce an immune response.

In recent years it has been shown that antibodies to glycolipids are detectable in MS patients', that such antibodies can demyelinate cerebellar tissue cultures ${ }^{2}$, that T-cell reactivity to glycolipids is present in MS patients ${ }^{3}$, and relatively specific to this disease $^{4}$, and that rabbits inoculated with glycolipids succumb to a demyelinating encephalomyelitis ${ }^{5}$. Thus it is clear that anti-glycolipid activity is of importance in demyelinating disease.

The question is, how does this antiglycolipid activity arise? One possibility, as we have suggested previously ${ }^{6}$, centres on the possibility that budding viruses can initiate an immune response to glycolipids. We have shown that infection of mice with the budding, avirulent RNA, Semliki Forest virus induces a $\mathrm{T}$-cell dependent demyelinating encephalomyelitis $^{7}$, although it is not yet clear whether the $\mathrm{T}$-cells responsible for the demyelination react against viral or self antigens on the surface of central nervous system (CNS) cells and myelin. The virus replicates in CNS cells including oligodendrocytes, host-cell glycolipids are incorporated into the viral envelope ${ }^{8}$, and these glycolipds are accessible to antibodies ${ }^{9}$. Infection with the virus and inoculation of inactivated brain derived virus both give rise to anti-glycolipid antibody (S. Amor and H.E.W., unpublished observations).

It is possible that several neurotropic budding viruses have the ability to replicate in the same CNS cell-type (for example oligodendrocytes) and on budding from this cell incorporate into their viral envelope the same host-cell glycolipid (for example galactocerebroside). It is possible that such a glycolipid (normally a hapten), associated on the surface of the virus with the 'foreign' carrier antigens of the viral proteins could be antigenic. In an MS susceptible individual, infection of the CNS with one of the many possible enveloped viruses could trigger an antiglycolipid immune reaction, leading to demyelination and a first attack of MS. Subsequent relapses in MS, already known to have an association with intercurrent infection, could result from restimulation of the same anti-glycolipid autoimmune response following CNS infection with other enveloped viruses.

Perhaps in the search for the cause of MS, more attention should be given to the possibility that viral envelope glycolipids could induce an immune mediated demyelination, and by the immunologists to the possibility that this may trigger an autoimmune encephalomyelitis.

J.K. FAZAKERLEY H.E. WeBB

Neurovirology Research Unit,

The Rayne Institute,

St Thomas' Hospital,

London SE1 7EH, UK

1. Arnon, R. et al. J. neurol. Sci. 26, 179 (1980).

2. Dubois-Dalcq, M., Niedieck, B. \& Buyse, M. Path. eur. 5, $331(1970)$.

Offner, H., Konat, G. \& Sela, B.A. J. neurol. Sci. 52, 279 (1981)

4. Ilyas, A.A. \& Davison. A.N. J. neurol. Sci. 59, 85 (1983).

Konat, G. et al. Acta neurol. Scand. 66, 568 (1982)

6. Webb, H.E. \& Fazakerley, J.K. Neuropathol. appl Neurobiol. 10, 1 (1984).

7. Fazakerley, J.K., Amor, S. \& Webb, H.E. Clin. exp Immun. 52, 115 (1983)

Webb, H.E., Mehta. S., Leibowitz, S. \& Gregson, N.A. Neuropathol appl. Neurobiol. 10, 77 (1984).

9. Evans, N.R. \& Webb, J. neurol. Sci. (in the press)

\section{Ventral activation process in insect oocytes}

SIR-In a recent News and Views article Woodland and Jones reviewed genetic evidence of an active ventral region in establishing the dorsoventral polarity and hence pattern in Drosophila eggs ${ }^{1}$. In particular, various recessive - and presumably loss of function - genes are all dorsalizing: not only the genes so lucidly discussed by Woodland and Jones but also the remarkable $K 10$ gene. Unlike the other dorsal mutants, $K 10$ acts on the growing oocyte so as to largely symmetrize the early movements of its follicle cells. Thus it produces an egg largely devoid of dorsoventral asymmetry in its shape and shell as well as dorsalizing its embryo $^{2,3}$. Thus lack of $K 10$ function seems to disable the mechanisms for establishing a dorsoventral gradient. Since it does so by disabling some ventrally localized process, it acutely raises the question of just what this ventral process is.

A clue to this was provided by Kunkel in a study of an analogous growth stage of the cockroach oocyte ${ }^{4}$. He has discovered the presence of large, steady electrical (and thus ionic) currents entering its ventral region. Comparable steady currents rather generally enter regions of developmental action, and in the best studied case - that of the fucoid egg they seem to establish this region by raising free calcium there ${ }^{5,6}$. So Kunkel's observations suggest that a key early event in Drosophila pattern formation is an influx of cations into its ventral region which raises free calcium there, activating gene products like those of Toll.

This hypothesis also suggests an explanation for the rather puzzling fact that $\mathrm{Toll}^{+}$ cytoplasm from anywhere in the egg nevertheless induces a ventral region near the site of injection. Perhaps the $\mathrm{Toll}^{+}$ precursor postulated by Anderson et al. ${ }^{7}$ is artificially activated during transfer by contact with the high calcium found in the perivitelline fluid of the Drosophila egg $^{8}$. This subsidiary hypothesis predicts that the ability of $\mathrm{Toll}^{+}$cytoplasm to rescue Toll eggs would be greatly reduced if it were transferred in a way which somehow avoided any transient rise in its free calcium concentration. This postulated artefactual rise would be analogous to the rise in calcium which can destroy the so-called primary cytostatic factor in the cytoplasm of frog eggs'.

In any case, the main hypothesis predicts the presence of a free calcium gradient across the dorso-ventral axis of the growing insect oocyte with free calcium high along its ventral face. This predicted ventral high calcium zone would be analogous to that already seen - with the aid of aequorin - at the vegetal pole of the early medaka fish egg. ${ }^{10}$.

Marine Biological Laboratory,

L.F. JAFFE

Woods Hole,

Massachusetts 02543, USA

Woodland, H.R. \& Jones, E.A. Nature 319, $261-262$ (1986)

2. Wieschaus, E. in Cell Lineage. Stem Cells and Cell Determination (ed. Le Douarin, N.) 291 - 302 (Elsevier, Amsterdam, 1979).

3. Haenlin, M. Stellar, H., Pirrotta, V. \& Mohier, E. Cell 40, $827-837(1985)$

4. Kunkel, J.G in Ionic Currents in Development (ed. Nuccitelli, R.) 165 - 172 (Liss. New York, 1986)

5. Jaffe, L.F. in Membrane Transduction Mechanisms (eds Cone, R.A. \& Dowling. J.E.) $199-231$ (Raven, New York, 1979).

6. Brownlee, C. \& Woods, J.W. Nature 320, 624-626 (1986) . Anderson, K.V., Jurgens, G. \& Nusslein-Volhard, C. Cell 42, 799-789 (1985)

Van der Meer, J.M. \& Jaffe, L.F. Devl Biol. 95, 249 - 252 (1983)

9. Masui, Y.. Meyerhof, P.G. \& Miller, M.A. Symp. Soc. dev. Biol. 38, $235-256(1980)$

10. Jaffe, L.F. CIBA Symp. 122 (in the press) 